\title{
Simplified Unitarity Triangles for the Lepton Sector
}

\author{
James D. Bjorken, ${ }^{1, *}$ P. F. Harrison, ${ }^{2, \dagger}$ and W. G. Scott ${ }^{3, \ddagger}$ \\ ${ }^{1}$ Stanford Linear Accelerator Center, Stanford University, Stanford, California 94309, USA \\ ${ }^{2}$ Department of Physics, University of Warwick, Coventry CV4 7AL, UK \\ ${ }^{3}$ CCLRC Rutherford Appleton Laboratory, Chilton, Didcot, Oxon OX11 OQX, UK
}

(Dated: October 31, 2018)

\begin{abstract}
Encouraged by the latest SNO results, we consider the lepton mixing matrix in the approximation that the $\nu_{2}$ mass eigenstate is trimaximally (democratically) mixed. This suggests a new parameterization of the remaining mixing degrees of freedom, which eschews mixing angles, dealing instead, directly with the complex parameter $U_{e 3}$ of the mixing matrix. Unitarity triangles then take a particularly simple form, which we hope will faciltate comparison with experiment.
\end{abstract}

PACS numbers: 14.60.Pq, 11.30.Er, 11.30.Hv

Recent years have seen huge advances in our knowledge of the properties of neutrinos. Most recently, SNO $[1,2]$ has provided the best evidence for neutrino flavour change, which, coupled with evidence from atmospheric neutrinos [3, 4], reactors [5-7] and accelerator experiments [8], has enabled the basic form of the MNS [9] lepton mixing matrix, $U$, to be determined [10].

Atmospheric neutrino data $[3,4]$, together with $\mathrm{K} 2 \mathrm{~K}$ $[8]$ and reactor data $[5,6]$, give (at $68 \% \mathrm{CL}$ ) [10]:

$$
\left|U_{e 3}\right|^{2} \lesssim 0.013 \quad\left|U_{\mu 3}\right|^{2}=0.50 \pm 0.11
$$

Therefore $\left|U_{\tau 3}\right|^{2} \approx 0.50 \pm 0.11$, implying that

$$
\left|U_{e 3}\right|^{2} \approx 0 \quad\left|U_{\mu 3}\right| \approx\left|U_{\tau 3}\right| \approx \frac{1}{\sqrt{2}}
$$

We may choose the phases of $\nu_{\mu}$ and $\nu_{\tau}$ such that to a good approximation

$$
\nu_{3}=\frac{1}{\sqrt{2}}\left(\nu_{\mu}-\nu_{\tau}\right)
$$

Unitarity then implies that, with the above choice of phases,

$$
U_{\mu 2} \approx U_{\tau 2} \quad U_{\mu 1} \approx U_{\tau 1}
$$

ie. approximate $\mu-\tau$ symmetry [11-13]. From the analysis of solar neutrino data [14-18], especially from SNO [1, 2], we have (at $68 \% \mathrm{CL}$ ):

$$
\left|U_{e 2}\right|^{2}=0.31 \pm 0.04
$$

implying

$$
\left|U_{e 2}\right| \approx\left|U_{\mu 2}\right| \approx\left|U_{\tau 2}\right| \approx \frac{1}{\sqrt{3}} .
$$

The phases of $\nu_{2}$ and $\nu_{e}$ can be chosen such that

$$
\nu_{2}=\frac{1}{\sqrt{3}}\left(\nu_{e}+\nu_{\mu}+\nu_{\tau}\right)
$$

Unitarity now fixes the remaining three MNS matrix elements up to their overall phase, which may be chosen so that

$$
\nu_{1}=\frac{1}{\sqrt{6}}\left(2 \nu_{e}-\nu_{\tau}-\nu_{\mu}\right) .
$$

Both the relative precision, and the absolute precision, in the determination of $\left|U_{e 2}\right|^{2}$ in Eq. (5) is better than that of $\left|U_{\mu 3}\right|^{2}$ in Eq. (1), making $\left|U_{e 2}\right| \approx \frac{1}{\sqrt{3}}$ currently the best-determined of the MNS matrix elements.

Equations (3), (7) and (8) together define the tribimaximal mixing texture [19-21], which we may take to be the leading approximation to the lepton mixing matrix. This texture is clearly evocative of symmetries at work. Taking the neutrino flavour eigenstates to define the orientation of a cube, the $\nu_{2}$ eigenstate, Eq. (7), lies along the body diagonal of the cube, while the $\nu_{3}$ mass eigenstate lies in the plane of the $\nu_{\mu}-\nu_{\tau}$ face, at $45^{\circ}$ to the $\nu_{\mu}$ state. It may be remarked that the same mixing matrix elements also occur as the $M=0$ subset of the $j \times j=1 \times 1$ set of Clebsch-Gordan coefficients.

An extensive future experimental neutrino program is being planned [22-24] which will refine the tribimaximal picture outlined above. The current situation for neutrino physics and the MNS matrix appears analogous to that earlier for $B$ physics and the CKM matrix, in which the leading approximation to the matrix was established experimentally, long before its smallest elements were determined. In that case, the Wolfenstein parametrization has become widely adopted [25]. This approximate parameterization avoids the introduction of mixing angles, dealing instead directly with the elements of the mixing matrix. Visualisation of its complex elements has been facilitated with the help of "unitarity triangles" [26-32], which, in their normalised form [33] use only two parameters. It is our purpose here to propose a simple two-parameter approximation for the MNS mixing in the lepton sector. It is motivated by the phenomenological success of tribimaximal mixing, which it takes as a starting point, but does not in fact depend upon this mixing form being exact, or even theoretically consequential. 
In the CKM case, phases are chosen such that the diagonal elements, as well as the two elements in the row above the diagonal, are chosen real and positive. Then unitarity controls the remaining elements, with the free variable of greatest importance being $V_{u b}$. The values of the two elements in the row below the diagonal are insensitive to details, while the remaining one, $V_{t d}$, is related to $V_{u b}$ via the well-known unitarity triangle.

For the case of the MNS matrix, the optimal choice of phases is different because the mixing pattern is different. The most striking feature of the data is arguably the near-democratic mixing of the $\nu_{2}$ mass eigenstate, as expressed in Eq. (6). As we show later, even for subdominant oscillation phenomena, it is a robust approximation to assume exact trimaximal mixing for the $\nu_{2}$ column. A natural choice of phases is then to require the $\nu_{2}$ column to be real and positive. The remaining elements are then controlled by $U_{e 3}$ (the analogue of $V_{u b}$ ), which is again small, and again vital to the unresolved issue of $C P$ violation, this time within the lepton sector.

We construct a generalisation [34-36] of the tribimaximal form, consistent with the above considerations:

$$
\begin{aligned}
U & \simeq\left(\begin{array}{ccc}
\frac{2}{\sqrt{6}} & \frac{1}{\sqrt{3}} & 0 \\
-\frac{1}{\sqrt{6}} & \frac{1}{\sqrt{3}} & \frac{1}{\sqrt{2}} \\
-\frac{1}{\sqrt{6}} & \frac{1}{\sqrt{3}} & -\frac{1}{\sqrt{2}}
\end{array}\right)\left(\begin{array}{ccc}
C & 0 & \sqrt{\frac{3}{2}} U_{e 3} \\
0 & 1 & 0 \\
-\sqrt{\frac{3}{2}} U_{e 3}^{*} & 0 & C
\end{array}\right) \\
& =\left(\begin{array}{ccc}
\frac{2}{\sqrt{6}} C & \frac{1}{\sqrt{3}} & U_{e 3} \\
-\frac{1}{\sqrt{6}} C-\frac{\sqrt{3}}{2} U_{e 3}^{*} & \frac{1}{\sqrt{3}} & \frac{1}{\sqrt{2}} C-\frac{1}{2} U_{e 3} \\
-\frac{1}{\sqrt{6}} C+\frac{\sqrt{3}}{2} U_{e 3}^{*} & \frac{1}{\sqrt{3}} & -\frac{1}{\sqrt{2}} C-\frac{1}{2} U_{e 3}
\end{array}\right)
\end{aligned}
$$

where

$$
C=\sqrt{1-\frac{3}{2}\left|U_{e 3}\right|^{2}} \simeq 1
$$

The matrix in Eq. (9) is exactly unitary, by construction. The need for the factor $\sqrt{\frac{3}{2}}$ in the second matrix in the expansion of Eq. (9) is to keep $U_{e 3}$ itself as the operative parameter in the resulting product. Dropping terms of order $\left|U_{e 3}\right|^{2}$ for simplicity (ie. setting $C=1$ ), we obtain the approximation

$$
U \simeq\left(\begin{array}{ccc}
\frac{2}{\sqrt{6}} & \frac{1}{\sqrt{3}} & U_{e 3} \\
-\frac{1}{\sqrt{6}}-\frac{\sqrt{3}}{2} U_{e 3}^{*} & \frac{1}{\sqrt{3}} & \frac{1}{\sqrt{2}}-\frac{1}{2} U_{e 3} \\
-\frac{1}{\sqrt{6}}+\frac{\sqrt{3}}{2} U_{e 3}^{*} & \frac{1}{\sqrt{3}} & -\frac{1}{\sqrt{2}}-\frac{1}{2} U_{e 3}
\end{array}\right) .
$$

In our parameterization, $\left|U_{e 3}\right|$ corresponds exactly to the same quantity in the standard (PDG) parameterization [33]:

$$
\left|U_{e 3}\right|=\sin \theta_{13}
$$

while our phase convention for $U_{e 3}$ differs only slightly from the usual one. This phase is fixed by the construction in Eq. (9) such that $\delta^{\prime} \equiv-\operatorname{Arg}\left(U_{e 3}\right)$ is given by

$$
\begin{aligned}
\sin \delta^{\prime} & =\sin 2 \theta_{23} \sin \delta \\
& \simeq \sin \delta
\end{aligned}
$$

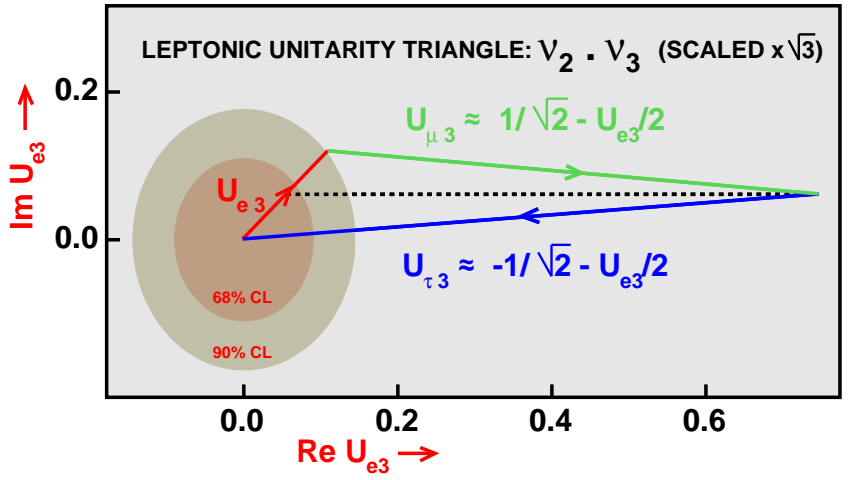

FIG. 1: The " $\nu_{2} . \nu_{3}$ " unitarity triangle representing the orthogonality of the $\nu_{2}$ and $\nu_{3}$ mass eigenstates (the dashed line bisects the $U_{e 3}$ side, is real and has length $\frac{1}{\sqrt{2}} C$ ). The triangle has been scaled by a factor $\sqrt{3}$ so that the sides are equal to the components of the $\nu_{3}$ column of the lepton mixing matrix. The error-ellipse indicates the allowed range of $\left|U_{e 3}\right|$ : the vertical error is given by the reactor bound $[5,6]$, while the horizontal error was obtained by combining this with the implied constraint from the experimental value of $\left|U_{\mu 3}\right|$ (Eq. (1)), under the assumption of exact trimaximal mixing of the $\nu_{2}$ mass eigenstate.

(this explains our choice of sign for the $\nu_{3}$ column). Exact equivalence of these two phase definitions is obtained in the two special cases: $\operatorname{Re}\left(U_{e 3}\right)=0$ or $\operatorname{Im}\left(U_{e 3}\right)=0$.

$C P$ violation is, of course, governed by $\operatorname{Im}\left(U_{e 3}\right)$. We have for Jarlskog's $C P$-violating observable [37, 38]:

$$
J_{C P}=\frac{C \operatorname{Im}\left(U_{e 3}\right)}{3 \sqrt{2}} \simeq \frac{\operatorname{Im}\left(U_{e 3}\right)}{3 \sqrt{2}} .
$$

The parameter $\operatorname{Re}\left(U_{e 3}\right) \simeq \sqrt{2}\left(\frac{\pi}{4}-\theta_{23}\right)$ clearly violates $\mu-\tau$ reflection symmetry (simultaneous interchange of $\mu$ and $\tau$ flavour labels and a $C P$ transformation [12]).

In the $C P$-violating case, leptonic unitarity triangles [39-41] may be constructed using the orthogonality of pairs of columns (or rows) of the mixing matrix. With our parameterization, Eqs. (9)-(11), most of the triangles become especially simple, eg. the " $\nu_{2} . \nu_{3}$ " triangle shown in Fig. 1, expressing the orthogonality of the $\nu_{2}$ and $\nu_{3}$ columns. Its sides are all proportional to the elements of the $\nu_{3}$ state (with a common factor $1 / \sqrt{3}$ removed by rescaling). This is perhaps the most useful of the six possible triangles, as one of its sides is simply proportional to $U_{e 3}$ itself, and is hence the leptonic analogue of the most commonly used "d.b" triangle of the quark sector.

The triangle expressing the orthogonality of the $\nu_{1}$ and $\nu_{2}$ columns has a similar simplification to that of the $\nu_{2} \cdot \nu_{3}$ triangle discussed above, its sides all being proportional to the elements of the $\nu_{1}$ mass eigenstate. It may be constructed from a (real) line of length $U_{e 1}=\frac{2}{\sqrt{6}} C$, bisected by a (complex) line given by $\frac{\sqrt{3}}{2} U_{e 3}$ whose end defines the third vertex. The triangle expressing the orthogonality of the $\nu_{1}$ and $\nu_{3}$ columns is the least degenerate 
of the three column-based triangles, but has no special simplification, in general, here.

The three triangles representing orthogonality of the rows of the MNS matrix are now similarly determined entirely in terms of $U_{e 3}$. They each share one angle with each of the column-wise triangles. Note that with $\nu_{2}$ exactly trimaximally mixed each of these row-based triangles stands on a common real base of length $\frac{1}{3}$, which could be rescaled to unity if desired.

Of course, the rephasing invariance of observables means that only the triangles' opening angles, sidelengths, and areas have physical significance (see below), their orientation being irrelevant. Symmetries are reflected in the shapes of the triangles, eg. with exact $\mu-\tau$ reflection symmetry, all three column-based triangles are isosceles (their sides carry lepton flavour indices), while the $\nu_{e} . \nu_{\tau}$ and $\nu_{e} \cdot \nu_{\mu}$ triangles become congruent to each other. If $C P$ is conserved, each triangle reduces to a line.

We turn now to the use of the triangles in the phenomenology of leptons, especially neutrino oscillations. The sides of the row-based triangles are given by complex products of MNS matrix elements, $U_{\alpha i} U_{\beta i}^{*}$, where the flavour labels $\alpha$ and $\beta$ define the triangle, and the mass index $i$, labels the side. These triangle sides are the (complex) magnitudes of the three sub-amplitudes which mediate neutrino oscillations between flavour $\alpha$ and flavour $\beta$, one for each neutrino mass eigenstate, $i$ :

$$
\begin{aligned}
\left|A\left(\nu_{\alpha} \rightarrow \nu_{\beta}\right)\right| & =\left|\sum_{i=1,3} U_{\alpha i} U_{\beta i}^{*} \mathrm{e}^{\left(-i m_{i}^{2} L / 2 E\right)}\right| \\
& =2\left|U_{\alpha i} U_{\beta i}^{*} \sin \Delta_{i k}+U_{\alpha j} U_{\beta j}^{*} \sin \Delta_{j k} \mathrm{e}^{i \Delta_{i j}}\right|
\end{aligned}
$$

$(\alpha \neq \beta, i \neq j \neq k)$, where $\Delta_{i j} \equiv\left(m_{i}^{2}-m_{j}^{2}\right) L / 4 E$, $L$ is the propagation distance, $E$ is the neutrino energy and $m_{i}$ is the mass of $\nu_{i}$. Squaring Eq. (16) to obtain appearance probabilities, we see that the triangles' side-lengths, $\left|U_{\alpha i} U_{\beta i}^{*}\right|$, and their external angles, $\phi_{\gamma k} \equiv \operatorname{Arg}\left(U_{\alpha i} U_{\beta i}^{*} U_{\alpha j}^{*} U_{\beta j}\right),(\gamma \neq \alpha$ etc. $)[46]$, appear explicitly (see eg. Eq. (21) below). With our parameterization, $U_{\alpha 2} U_{\beta 2}^{*}=1 / 3$ for all flavour-pairs, $\alpha$, $\beta$, while other side-lengths become simple functions of $U_{e 3}$.

The $C P$ parameter, $J_{C P}=\left|U_{\alpha i} U_{\beta i}^{*}\right|\left|U_{\alpha j} U_{\beta j}^{*}\right| \sin \phi_{\gamma k}$, $\forall \gamma, k$, equals twice the area of each (un-rescaled) triangle.
For the triangle in Fig. 1, we find using Eq. (15):

$$
\sin \phi_{\alpha 1}=\frac{C \operatorname{Im}\left(U_{e 3}\right)}{\sqrt{2}\left|U_{\beta 3}\right|\left|U_{\gamma 3}\right|} \simeq \frac{\operatorname{Im}\left(U_{e 3}\right)}{\sqrt{2}\left|U_{\beta 3}\right|\left|U_{\gamma 3}\right|}
$$

with $\alpha \neq \beta \neq \gamma$. Expressions may similarly be obtained for the angles of all the other triangles.

Expressions for neutrino oscillation survival probabilities involve directly the moduli-squared of MNS matrix elements, $\left|U_{\alpha i}\right|^{2}$. Here again, $\left|U_{\alpha 2}\right|^{2}=1 / 3$ for all flavours $\alpha$. For $i=3$, these are the side lengths of the $\nu_{2} \cdot \nu_{3}$ triangle of Fig. 1 which (for non-trivial cases) are given by:

$$
\begin{aligned}
\left|U_{\alpha 3}\right|^{2} & =\frac{1}{2}-\frac{\left|U_{e 3}\right|^{2}}{2} \mp \frac{C \operatorname{Re}\left(U_{e 3}\right)}{\sqrt{2}} \\
& \simeq \frac{1}{2} \mp \frac{\operatorname{Re}\left(U_{e 3}\right)}{\sqrt{2}}
\end{aligned}
$$

where the upper(lower) signs correspond to $\alpha=\mu(\tau)$, respectively (the approximate form may be obtained directly by inspection of Fig. 1). The analogous result for $i=1$ is obtained from the $\nu_{1} \cdot \nu_{2}$ triangle as:

$$
\begin{aligned}
\left|U_{\alpha 1}\right|^{2} & =\frac{1}{6}+\frac{\left|U_{e 3}\right|^{2}}{2} \pm \frac{C \operatorname{Re}\left(U_{e 3}\right)}{\sqrt{2}} \\
& \simeq \frac{1}{6} \pm \frac{\operatorname{Re}\left(U_{e 3}\right)}{\sqrt{2}}
\end{aligned}
$$

A new generation of experiments is being planned and built to address the question of non-leading neutrino oscillations [22-24]. The description of the observables at these new experiments is usefully presented as a series expansion [42] in the two small quantities:

$$
\alpha \equiv \frac{\Delta_{21}}{\Delta_{31}} \simeq 0.026,0.021 \lesssim \alpha \lesssim 0.036(90 \% \mathrm{CL}),
$$

(describing the hierarchy of mass-squared differences), and $\left|U_{e 3}\right|=\sin \theta_{13} \lesssim 0.17$ (90\% CL), as a function of the standard parameters. In terms of our parameterization, we find a considerable simplification. To leading (second) order in small quantities, the $\nu_{e}$ appearance probability in a long baseline muon neutrino beam is (in vacuum):

$$
P\left(\nu_{\mu} \rightarrow \nu_{e}\right) \simeq 2\left|U_{e 3}\right|^{2} \sin ^{2} \Delta_{31}+\frac{4}{9} \alpha^{2} \Delta_{31}^{2}+\frac{8 \alpha\left|U_{e 3}\right|}{3 \sqrt{2}} \Delta_{31} \sin \Delta_{31} \cos \left(\Delta_{31}+\delta^{\prime}\right)
$$

where the $C P$-violating phase $\delta^{\prime} \simeq \delta \simeq-\phi_{\tau 1}$ at this order of approximation (see Fig. 1 and Eqs. (13)-(14)). The electron anti-neutrino disappearance probability at a (not too distant) reactor experiment is given, again to leading (second) order in small quantities, by:

$$
1-P\left(\bar{\nu}_{e} \rightarrow \bar{\nu}_{e}\right) \simeq 4\left|U_{e 3}\right|^{2} \sin ^{2} \Delta_{31}+\frac{8}{9} \alpha^{2} \Delta_{31}^{2} .
$$

Although Eqs. (21) and (22) have been derived under the 
assumption of exact trimaximal mixing of the $\nu_{2}$ mass eigenstate, it can be shown that they are still valid at second order in small quantities, even in the case that this assumption is broken by small perturbations. This justifies our proposal of Eq. (11) as a viable approximate parameterization of the MNS matrix.

As well as oscillation phenomenology, the row-based triangles play a role in (lepton number violating) radiative decays of charged leptons, while the column-based ones play an analogous role in radiative decays of neutrinos, although admittedly, these are unlikely to play a major role in phenomenology, as they are all highly suppressed in the Standard Model [43].

Clearly, neutrino oscillation experiments have come a long way since one could countenance the idea of trimaximal mixing for all three neutrino species [19, 44, 45]. There is today however, still the real prospect that the $\nu_{2}$ mass eigenstate is indeed trimaximally mixed, and this hints at deeper symmetries beyond the Standard Model. While it is important to test experimentally whether the $\nu_{2}$ mass eigenstate is exactly trimaximally mixed, we have argued that this assumption anyway provides a simplifying and illustrative approximation to the phenomenology of neutrino oscillations.

This work was supported by the UK Particle Physics and Astronomy Research Council (PPARC), and in part, by the US. Department of Energy contract DE-AC0276SF00515. Two of us (PFH and JDB) acknowledge the hospitality of the Centre for Fundamental Physics (CfFP) at CCLRC Rutherford Appleton Laboratory.

* bjorken@slac.stanford.edu

$\dagger$ p.f.harrison@warwick.ac.uk

\#.g.scott@rl.ac.uk

[1] B. Aharmim et al. (SNO), Phys. Rev. C72, 055502 (2005), nucl-ex/0502021.

[2] S. N. Ahmed et al. (SNO), Phys. Rev. Lett. 92, 181301 (2004), nucl-ex/0309004.

[3] Y. Ashie et al. (Super-Kamiokande), Phys. Rev. D71, 112005 (2005), hep-ex/0501064.

[4] M. C. Sanchez et al. (Soudan 2), Phys. Rev. D68, 113004 (2003), hep-ex/0307069.

[5] M. Apollonio et al., Eur. Phys. J. C27, 331 (2003), hepex/0301017.

[6] F. Boehm et al., Phys. Rev. D64, 112001 (2001), hepex/0107009.

[7] K. Eguchi et al. (KamLAND), Phys. Rev. Lett. 90, 021802 (2003), hep-ex/0212021.

[8] M. H. Ahn et al. (K2K), Phys. Rev. Lett. 90, 041801 (2003), hep-ex/0212007.

[9] Z. Maki, M. Nakagawa, and S. Sakata, Prog. Theor. Phys. 28, 870 (1962).

[10] A. Strumia and F. Vissani, Nucl. Phys. B726, 294 (2005), hep-ph/0503246.

[11] C. S. Lam, Phys. Lett. B507, 214 (2001), hep$\mathrm{ph} / 0104116$.
[12] P. F. Harrison and W. G. Scott, Phys. Lett. B547, 219 (2002), hep-ph/0210197.

[13] W. Grimus and L. Lavoura, Phys. Lett. B579, 113 (2004), hep-ph/0305309.

[14] S. Fukuda et al. (Super-Kamiokande), Phys. Lett. B539, 179 (2002), hep-ex/0205075.

[15] J. N. Abdurashitov et al. (SAGE), J. Exp. Theor. Phys. 95, 181 (2002), astro-ph/0204245.

[16] W. Hampel et al. (GALLEX), Phys. Lett. B447, 127 (1999)

[17] M. Altmann et al. (GNO), Phys. Lett. B490, 16 (2000), hep-ex/0006034.

[18] B. T. Cleveland et al., Astrophys. J. 496, 505 (1998).

[19] P. F. Harrison, D. H. Perkins, and W. G. Scott, Phys. Lett. B458, 79 (1999), hep-ph/9904297.

[20] P. F. Harrison, D. H. Perkins, and W. G. Scott, Phys. Lett. B530, 167 (2002), hep-ph/0202074.

[21] P. F. Harrison and W. G. Scott (2004), hep-ph/0402006.

[22] Y. Hayato (T2K), Nucl. Phys. Proc. Suppl. 143, 269 (2005).

[23] D. S. Ayres et al. (NOvA) (2004), hep-ex/0503053.

[24] M. Goodman, Nucl. Phys. Proc. Suppl. 145, 186 (2005), hep-ph/0501206.

[25] L. Wolfenstein, Phys. Rev. Lett. 51, 1945 (1983).

[26] L.-L. Chau and W.-Y. Keung, Phys. Rev. Lett. 53, 1802 (1984).

[27] J. D. Bjorken, Phys. Rev. D39, 1396 (1989).

[28] C. Jarlskog and R. Stora, Phys. Lett. B208, 268 (1988).

[29] J. L. Rosner, A. I. Sanda, and M. P. Schmidt (1987), Proceedings of the Workshop on High Sensitivity Beauty Physics, Batavia, IL.

[30] C. Hamzaoui, J. L. Rosner, and A. I. Sanda (1987), ibid.

[31] G. Branco and L. Lavoura, Phys. Lett. B208, 123 (1988).

[32] J. A. Aguilar-Saavedra and G. C. Branco, Phys. Rev. D62, 096009 (2000), hep-ph/0007025.

[33] S. Eidelman et al. (Particle Data Group), Phys. Lett. B592, 1 (2004).

[34] P. F. Harrison and W. G. Scott, Phys. Lett. B535, 163 (2002), hep-ph/0203209.

[35] P. F. Harrison and W. G. Scott, Phys. Lett. B557, 76 (2003), hep-ph/0302025.

[36] P. F. Harrison and W. G. Scott, Phys. Lett. B594, 324 (2004), hep-ph/0403278.

[37] C. Jarlskog, Z. Phys. C29, 491 (1985).

[38] C. Jarlskog, Phys. Rev. Lett. 55, 1039 (1985).

[39] Y. Farzan and A. Y. Smirnov, Phys. Rev. D65, 113001 (2002).

[40] Z. Xing and H. Zhang, Phys. Lett. B618, 131 (2005).

[41] H. Zhang and Z. Xing, Eur. Phys. J. C41, 143 (2005).

[42] E. K. Akhmedov, R. Johansson, M. Lindner, T. Ohlsson, and T. Schwetz, JHEP 04, 078 (2004), hep-ph/0402175.

[43] F. Boehm and P. Vogel (1992), Physics of Massive Neutrinos, Cambridge University Press.

[44] P. F. Harrison, D. H. Perkins, and W. G. Scott, Phys. Lett. B349, 137 (1995).

[45] P. F. Harrison, D. H. Perkins, and W. G. Scott, Phys. Lett. B396, 186 (1997), hep-ph/9702243.

[46] The $\phi_{\alpha i}$ notation, serves equally for both sets of triangles: for row-based triangles, the index $\alpha$ defines the triangle representing the orthogonality of eigenstates $\nu_{\beta}$ and $\nu_{\gamma}$, while the index $i$ labels the triangle side opposite the angle in question. For column-based triangles, the interpretations of the first and second indices are exchanged. The same matrix of 9 angles describes both sets of triangles. 